\title{
Zoonotic Dirofilaria immitis and Dirofilaria repens infection in humans and an integrative approach to the diagnosis
}

\author{
Jairo Alfonso Mendoza-Roldan ${ }^{\mathrm{a}}$, Simona Gabrielli ${ }^{\mathrm{b}}$, Antonio Cascio ${ }^{\mathrm{c}}$, Ranju R.S. Manoj ${ }^{\mathrm{a}}$, \\ Marcos Antonio Bezerra-Santos ${ }^{\mathrm{a}}$, Giovanni Benelli ${ }^{\mathrm{d}}$, Emanuele Brianti ${ }^{\mathrm{e}}$, \\ Maria Stefania Latrofa ${ }^{a}$, Domenico Otranto ${ }^{\mathrm{a}, \mathrm{f}, *}$ \\ ${ }^{a}$ Department of Veterinary Medicine, University of Bari, Valenzano, Italy \\ b Dipartimento di Sanità Pubblica e Malattie Infettive Università di Roma "Sapienza", Roma, Italy \\ ${ }^{\mathrm{c}}$ Department of Health Promotion, Mother and Child Care, Internal Medicine and Medical Specialties- University of Palermo, 90127 Palermo, Italy \\ ${ }^{\mathrm{d}}$ Department of Agriculture, Food and Environment, University of Pisa, Pisa, Italy \\ e Department Veterinary Sciences, Polo Universitario Annunziata, Messina, Sicily, Italy \\ ${ }^{\mathrm{f}}$ Faculty of Veterinary Sciences, Bu-Ali Sina University, Hamedan, Iran
}

\section{A R T I C L E I N F O}

\section{Keywords:}

Dirofilariasis

Humans

Dogs

Canine heartworm disease

Vector-borne zoonosis

One Health

\begin{abstract}
A B S T R A C T
Dirofilariosis by Dirofilaria immitis and Dirofilaria repens is endemic in dogs from countries of the Mediterranean basin. Both species may infect humans, with most of the infected patients remaining asymptomatic. Based on the recent description of the southernmost hyperendemic European focus of heartworm disease in dogs from the Pelagie archipelagos, we performed a serological and molecular survey in human population of that area. Human blood samples were collected in the islands of Linosa $(n=101)$ and Lampedusa $(n=296)$ and tested by ELISA and molecular test for the detection of $D$. immitis and $D$. repens. Samples were also screened for filarioid-associated endosymbionts, Wolbachia sp. The seroprevalence of $D$. immitis and D. repens was, respectively, $7.9 \%$ and $3.96 \%$ in Linosa, and 7.77\% and 19.93\% in Lampedusa. Out of 397 human blood samples tested molecularly, 4 scored positive (1\%) for Dirofilaria spp. by qPCR (i.e., three for D. immitis and one for D. repens) and 6 (1.5\%) for Wolbachia. Of the qPCR positive for Dirofilaria spp., only D. repens was amplified by cPCR and was positive for Wolbachia. In the phylogenetic analysis, the sequence of Wolbachia detected in $D$. repens positive samples clustered along with other $C$ supergroup filarioids. Our results overlap with the recent prevalence data collected on dogs from the same area, where $D$. immitis is prevalent in Linosa and $D$. repens prevails in Lampedusa. Molecular detection of $D$. immitis in human blood is quite unusual considering that humans are dead-end hosts for dirofilarial infection and most of the human cases described so far in Europe were ascribed to D. repens. An integrative diagnostic approach using serum analysis and Wolbachia detection is also presented. In endemic areas for canine dirofilarioses humans are exposed to the infection, suggesting the importance of One Health approach in diagnosing, treating and controlling this zoonotic parasitosis.
\end{abstract}

\section{Introduction}

Dirofilaria immitis and Dirofilaria repens are widespread filarioids of dogs with occurrence in cats, ferrets, wild carnivores and humans, being both filarioids of major medical and veterinary concern, transmitted via the bite of infected mosquitoes (Dantas-Torres and Otranto, 2020; Panarese et al., 2020). Zoonotic cases of dirofilarioses have increased in the last decades paralleling the presence in endemic areas and also correlating with the new foci of infection in previously non endemic areas (Dantas-Torres and Otranto, 2020; Genchi and Kramer, 2020). Indeed, many factors have been implicated in the spreading of dirofilarioses, such as the relocation of dogs from endemic to non-endemic areas (e.g., from southern endemic areas of Europe to the northern ones) and the introduction of suitable allochthonous mosquito vectors (e.g., Aedes albopictus in the late '80s in Italy; Otranto et al., 2009). Humans act as dead-end hosts of both species of Dirofilaria, mainly $D$. repens in Europe and D. immitis in the USA (Dantas-Torres and Otranto, 2013). Human cases of $D$. immitis are scarce thought few reports were recorded

\footnotetext{
* Corresponding author at: University of Bari, Department of Veterinary Medicine, Str. prov. per Casamassima km 3 7..., 70010 Valenzano (Bari), Italy.

E-mail address: domenico.otranto@uniba.it (D. Otranto).
} 
in Europe (Avellis et al., 2011). Nonetheless, the zoonotic implication of these infections is relevant considering that soon after the inoculation by the mosquitoes, larvae may migrate throughout human tissues causing ocular, skin and pulmonary nodular lesions, often being erroneously diagnosed as pulmonary carcinomas (Gabrielli et al., 2021). Therefore, understanding the distribution and occurence of human cases of dirofilarioses is of relevance in differentially diagnosing the subcutaneous and/or pulmonary nodules or other suspected clinical presentations (Oliva et al., 2019). The global distribution of both Dirofilaria spp. overlaps with that of the mosquito vectors ( $>70$ species) belonging to the genera Aedes/Ochlerotatus, Anopheles and Culex (Eldridge and Edman, 2000). In Italy, Culex pipiens and Aedes albopictus are the main species implicated in the transmission of the infections (Otranto et al., 2009). Of note, their distribution, along with the lack of chemoprophylactic treatments in non-endemic regions have been considered as a major cause for the expansion of the infection, mainly in southern Italian regions (Otranto et al., 2009; Mendoza-Roldan et al., 2020).

Currently, hyperendemic foci of $D$. immitis infection in dogs are described in Apulia region (southern Italy) (Panarese et al., 2020a; Panarese et al., 2020b), as well as in the Pelagie archipelagos, with a prevalence of $58.9 \%$ for $D$. immitis and $7.9 \%$ for D. repens in Linosa and Lampedusa, respectively (Brianti et al., 2021). The latter finding represents the southernmost focus of the infection and triggered the scientific interest in investigating the exposure of asymptomatic patients living in a confined/close environment (i.e., Linosa and Lampedusa) where many interactions among humans, dogs and cats occur. This condition, which is typical of small islands, was further enhanced by limited movements of humans due to the COVID-19 pandemic restrictions. Under the above circumstances, the detection of Wolbachia has been suggested as a further test to refine the diagnosis of human dirofilarioses. These obligatory endosymbionts of filarioids play a major role in the pathogenesis of both acute and chronic filariasis; therefore, they have been targeted for the treatment and indirect diagnosis of these parasitosis (Turba et al., 2012; Manoj et al., 2021). Hence, the current study adopted an integrated approach including serology, molecular detection of filarioids and their endosymbionts Wolbachia.

\section{Materials and methods}

\subsection{Ethical statement}

The study on humans was conducted in accordance with ethical principles (Declaration of Helsinki) and the research protocol was approved by the Ethical Committee of the University Hospital of Palermo, Italy (n. 8/2020).

\subsection{Study site and sample collection}

Study sites have been described elsewhere (Brianti et al., 2021). Briefly, Lampedusa (20.2 sq km) and Linosa $(5.4 \mathrm{sq} \mathrm{km})$ are two small islands of the Pelagie archipelago, almost equidistant from Italian and Tunisian coasts and populated by nearly 300 and 6000 inhabitants, respectively. From July to October 2020, 346 human blood samples ( $n=245$ from Lampedusa and $n=101$ from Linosa) were collected from patients without any specific clinical signs and sent to the Parasitology unit of the Department of Veterinary Medicine, University of Bari (Italy) and Department of Public Health and Infectious Diseases, Sapienza University of Rome (Italy). Written informed consent was obtained from the participants and samples were received from human analysis laboratory (Lampedusa) or purposefully collected under the frame of this study (Linosa), for health check analyses.

\subsection{Detection of antibodies}

Human serum samples were tested to assess the exposure to $D$. immitis and $D$. repens by a home-made enzyme-linked immunosorbent assay (ELISA) based on somatic antigens of both Dirofilaria species (Simon et al., 1991; Tasić-Otašević et al., 2014). Antigens were isolated from worms obtained by necropsy of naturally infected dogs, washed, macerated and sonicated (three cycles of $70 \mathrm{kHz}, 30 \mathrm{~s}$ ) in sterile saline solution (Prieto et al., 1997). The homogenate was centrifuged at 16 , $000 \times \mathrm{g}$ for $30 \mathrm{~min}$. The supernatant was dialyzed against $0.01 \mathrm{M}$ PBS, $\mathrm{pH}$ 7.2. The protein concentration was measured (Bradford, 1976) and ELISA microplates were coated with antigens at final concentration of $0.8 \mu \mathrm{g} / \mu \mathrm{L}$. Following the protocols performed in previous surveys (Esteban-Mendoza et al., 2020; Otasevic et al. 2014), sera were tested in solid-phase ELISA at dilution 1:80 and 1:150 to detect anti-D. repens and anti-D. immitis IgG antibodies, respectively. Goat anti-human-IgGanti-human IgG $(\mathrm{H}+\mathrm{L})$ conjugated to horseradish peroxidase (Sigma-Aldrich, MO, USA) was used as a secondary antibody at 1:40000 dilution in both cases. Optical density (OD) was measured at $492 \mathrm{~nm}$ (Easy-Reader, Bio Rad, CA, USA). The cut-off points (OD = 1.12 for DiSA and $\mathrm{OD}=1.03$ for DrSA) was established by calculating the mean value +3 standard deviations (3SD) of 30 serum samples obtained from clinically healthy humans (negative controls).

\subsection{Molecular testing}

Genomic DNA (gDNA) was extracted from human blood samples using the commercial kit GenUPgDNA (Biotechrabbit GmbH, Hennigsdorf, Germany) according to the manufacturer's instructions. All samples were molecularly tested for Dirofilaria spp. by duplex real-time PCR (qPCR) as previously described (Latrofa et al., 2012). All DNA samples were tested in duplicate, and positive and negative controls were included in each qPCR run. The specificity of the qPCR assay was established by melting curve analysis as described in Latrofa et al. (2012). qPCR positive samples were amplified in conventional PCR (cPCR) using filarioid primers targeting 12S rRNA and cytochrome $c$ oxidase subunit 1 ( $\operatorname{cox} 1$ ) as previously described (Otranto et al., 2011). Blood and the filarioid positive samples were also tested for the endosymbiont, Wolbachia targeting 16S rRNA gene by qPCR (Willems et al., 1999) and cPCR (Werren and Windsor, 2000) respectively.

All amplified PCR products were visualized in 2\% agarose gel containing Gel Red®nucleic acid gel stain (VWR International PBI,Milan, Italy) and documented in Gel Logic 100 (Kodak, New York, NY, U.S.A.). The PCR products were purified and sequenced in both directions using the same primers, employing the Big Dye Terminator v.3.1 chemistry in a 3130 Genetic analyzer (Applied Biosystems, Foster City, CA, U.S.A.) in an automated sequencer (ABI-PRISM 377). Nucleotide sequences were edited, aligned and analyzed using BioEdit and compared with available sequences in the GenBank using Basic Local Alignment Search Tool (BLAST; http://blast.ncbi.nlm.nih.gov/Blast.cgi). All sequences were aligned using the ClustalW program (Larkin et al., 2007) and compared with those available in GenBank using the BLASTn tool (http://blast. ncbi.nlm.nih.gov/Blast.cgi).

\subsection{Phylogenetic analyses}

Wolbachia sequence targeting 16S rRNA gene obtained from the D. repens positive sample was phylogenetically analysed with the sequences available in the GenBank database. Phylogenetic relationships were inferred using Maximum Likelihood (ML) method based on Kimura 2-parameter model (Kimura, 1980) with discrete Gamma distribution $(+G)$ to model evolutionary rate differences among sites selected by best-fit model (Kumar et al., 2018). Evolutionary analyses were conducted on 1000 bootstrap replications using the MEGA X software (Tamura et al., 2013). Homologous sequences from Ehrlichia ruminantium and Ehrlichia chaffeensis were used as outgroups (NR074513, AF147752). 


\subsection{Statistical analysis}

Exact binomial 95\% confidence intervals (95\% CI) were established for $D$. immitis and $D$. repens positive proportions using GraphPad Prism v. 8.0.0 (GraphPad Software, San Diego, CA, USA). Thus, a weighted generalized linear model (GLZ) with a binomial distribution was used to model $D$. immitis or $D$. repens positivity (prevalence) in the two study islands, i.e., Lampedusa and Linosa. Following Mendoza-Roldan et al., (2020), a GLZ with two fixed factors was employed: $y=X \beta+\varepsilon$, where $y$ is the vector of the observation (i.e., the serological outcome: positive $=1$, negative $=0$ ), $X$ is the incidence matrix, $\beta$ is the vector of the fixed effects (i.e., the Dirofilaria study species and the surveyed island), and $\varepsilon$ is the vector of the random residual effects $(\alpha=0.05)$.

OD values for $D$. immitis and $D$. repens from serological tests (pooled data from both islands) were compared using the Wilcoxon's rank sum test $(\alpha=0.05)$. OD values from Dirofilaria spp.-positive samples from Lampedusa and Linosa (pooled $D$. immitis and $D$. repens data) were compared using the Wilcoxon's rank sum test $(\alpha=0.05)$. Furthermore, OD values for $D$. immitis and $D$. repens from serological tests were analyzed separately for each island relying to the Dunn's test with Bonferroni correction $(\alpha=0.05)$. All analyses were carried out using JMP ${ }^{\circledR} 11$ (SAS Institute, Cary, NC, USA), with a $p$-value $<0.05$ considered as statistically significant.

\section{Results}

Overall, 77 out of 397 patients (19.4\%, 95\% CI: 15.7-23.6) were positive to Dirofilaria spp. In particular, 31 were positive to $D$. immitis (7.8\%, 95\% CI: 5.5-10.9), 63 to D. repens (15.9\%, 95\% CI: 12.6-19.9) and 17 to both species (4.3\%, 95\% CI: 2.6-6.8) by serology and/or molecular tests. None of the individuals sampled reported specific medical conditions potentially compatible to dirofilariosis. Of the 31 patients positive to Dirofilaria spp. $6(1.5 \%)$ presented anti- Dirofilaria spp. antibodies titers higher than 2.0.

The overall prevalence of Dirofilaria spp. was significantly different among the two islands, being higher in Lampedusa over Linosa $\left(\chi^{2}=10.491 ;\right.$ d.f. $\left.=1 ; p=0.0112\right)$. Concerning the Dirofilaria species involved, analyzing pooled data from the two islands, the seropositivity for $D$. immitis was significantly higher than that for $D$. repens $\left(\chi^{2}=12.727\right.$; d.f. $=1 ; p<0.0001$ ).

However, analyzing Dirofilaria spp. seroprevalence data separately for each species and island, we noted significant differences $\left(\chi^{2}=30.698\right.$; d.f. $=3 ; p<0.0001$ ) in that, $D$. repens was significantly more prevalent in Lampedusa (59/296=; 19.93\%, 95\% CI: 15.7-24.97) than in Linosa ( $\mathrm{n}=4 / 101 ; 3.96 \%, 95 \%$ CI: 1.4-9.75) $\left(\chi^{2}=18.115 ; d . f .=1 ; p<0.0001\right)$. On the other hand, no significant differences were noted about $D$. immitis prevalence in Linosa ( $\mathrm{n}=8 / 101 ; 7.9 \%, 95 \%$ CI:3.7-15.2) over Lampedusa $(\mathrm{n}=23 / 296 ; 7.77 \%, 95 \%$ CI: $5.2-11.4) \quad\left(\chi^{2}=0.002 ; \quad\right.$ d.f. $=1$; $p=0.968)$.

Pooled data from the two Dirofilaria species showed higher OD median values characterizing the study samples from Lampedusa over those from Linosa $(Z=-2.204 ; d . f .=1 ; p=0.028)$ (Figure $1 \mathrm{a})$. On the other hand, data from both islands showed that the median OD values for $D$. immitis and for $D$. repens did not differ significantly $(Z=-0.519 ; d . f .=1$; $p=0.604$ ) (Figure $1 \mathrm{~b}-\mathrm{c}$ ). The minimum and maximum OD values for samples positive for $D$. immitis and $D$. repens in the two islands are detailed in Table 1.

Out of 397 human blood samples tested molecularly, 4 scored positive (1\%, 95\% CI: $0.4-2.6)$ for Dirofilaria spp. by qPCR. In particular, three samples were positive for $D$. immitis and one for $D$. repens by melting-curve analysis, showing specific melting peaks corresponding to $\mathrm{Tm}$ of $D$. immitis (i.e., mean $\mathrm{Tm}=75^{\circ} \mathrm{C}$ ) and $D$. repens (i.e., mean $\mathrm{Tm}=$ $\left.69^{\circ} \mathrm{C}\right)$. Six individuals (1.5\%; 95\% CI: 0.7-3.2) scored positive for $\mathrm{Wol}$ bachia by qPCR. In addition, from the screened samples that scored qPCR-positive for Dirofilaria spp., only $D$. repens was amplified by cPCR and also scored positive for Wolbachia. a
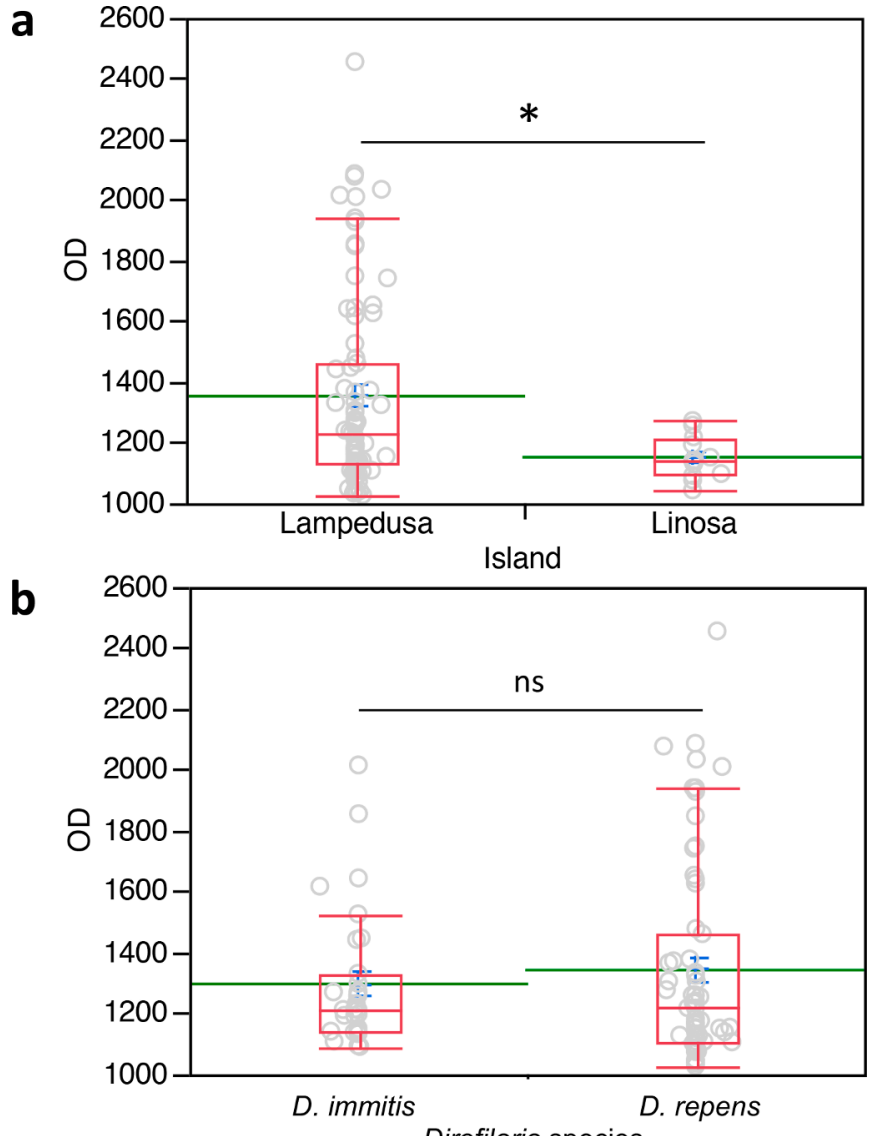

C

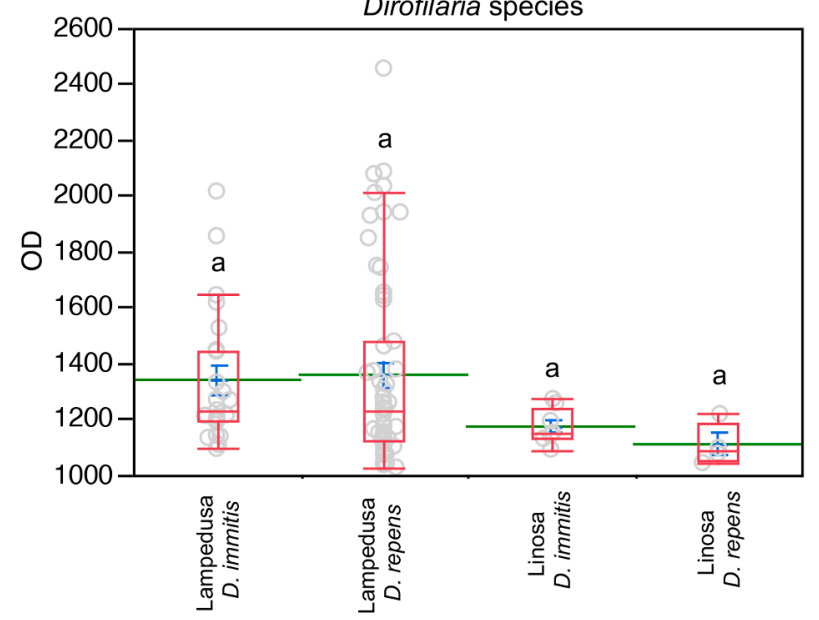

Fig. 1. OD values of serological tests for detecting Dirofilaria immitis and Dirofilaria repens in human blood samples from Lampedusa and Linosa islands, southern Italy. (a) Analyzing pooled data from the two Dirofilaria species, seroprevalence was higher in Lampedusa over Linosa. (b) Analyzing pooled data from the two islands, no significant differences were detected between the two Dirofilaria species. (c) OD data analyzed separately for each Dirofilaria species and study island. Red box plots indicate the median (solid line) within each box and the range of dispersion (lower and upper quartiles and outliers); means and standard errors are represented by green lines and blue T-bars, respectively. The asterisk indicates a significant difference $(p<0.05)$; ns $=$ not significant; the same letters each boxplot indicate the lack of significant differences among groups (a-b: Wilcoxon's rank sum test; c: Dunn's test with Bonferroni correction, $p>0.05)$. 


\section{Table 1}

Number and percentage (within brackets) of human blood samples scoring serological positive for $D$. immitis and $D$. repens in the Lampedusa and Linosa islands (southern Italy), along with the mean, minimum and maximum optical density (OD) recorded. Within each row, different superscript letters after prevalence numbers indicate that they differ significantly (GLZ, binomial distribution, $\mathrm{p}<0.05$ ).

\begin{tabular}{lllll}
\hline $\begin{array}{l}\text { Dirofilaria } \\
\text { species }\end{array}$ & $\begin{array}{l}\text { Lampedusa island } \\
\text { Number } \\
(\%)\end{array}$ & $\begin{array}{l}\text { Mean OD (nm) } \\
(\min -\max )\end{array}$ & $\begin{array}{l}\text { Linosa island } \\
\text { Number } \\
(\%)\end{array}$ & $\begin{array}{l}\text { Mean OD (nm) } \\
(\min -\max )\end{array}$ \\
\hline D. immitis & $8^{\mathrm{a}}(7.90 \%)$ & $1.340(1.095-$ & $23^{\mathrm{a}}$ & $1.173(1.092-$ \\
& & $2.016)$ & $(7.77 \%)$ & $1.273)$ \\
D. repens & $59^{\mathrm{a}}$ & $1.358(1.028-$ & $\begin{array}{l}3.96^{\mathrm{b}} \\
(4.0 \%)\end{array}$ & $1.100(1.044-$ \\
& $(19.93 \%)$ & $2.456)$ & $1.219)$ \\
\hline
\end{tabular}

In the phylogenetic tree of the 16S rRNA gene of Wolbachia the sequence detected in $D$. repens positive human clustered within the clade including other sequences of C supergroup Wolbachia from filarioids, supported by high bootstrap value (Figure 2). Representative Wolbachia sequence obtained in the current study was deposited in GenBank (accession number: MZ477525).

\section{Discussion}

Our results suggest that humans living in the islands of Lampedusa and Linosa (Pelagie archipelago) are exposed to both Dirofilaria spp., overlapping the distribution of dogs (Brianti et al., 2021). In particular, while the difference in prevalence of $D$. immitis infection in humans (7.8\%) in Linosa and Lampedusa is not statistically different, D. repens was more prevalent in Lampedusa. This picture overlaps the prevalence of both species in dogs from Lampedusa and Linosa, with $D$. repens recorded in Lampedusa and D. immitis in Linosa (Brianti et al., 2021). Conversely, the presence of human patients positive at the serology for D. repens in Linosa is suggestive of human traveling between the two islands.

The overall seroprevalence of $D$. repens and $D$. immitis in the human population sampled in this study is higher than those recently recorded elsewhere. For example, from a total of 450 patients tested in Romania and Moldova, $10.9 \%$ presented a positive response to antigen tests, with the seroprevalence only for $D$. immitis (i.e., $10.8 \%$ ) and only $0.2 \%$ for D. repens (Ciuca et al., 2018). Similarly, 5.11\% and $6.4 \%$ of tested patients in Mexico and in the Canary Islands, respectively, were seropositive for D. immitis (Cabrera et al., 2018; Zumaquero et al., 2020). $D$. repens is considered the main causative agent of human dirofilariosis in Europe (Otranto et al., 2013; Tasić-Otašević et al., 2015; Genchi and Kramer, 2020), which has been herein confirmed by the high seroprevalence (19.93\%) recorded for this species in Lampedusa. Conversely, this pattern has not been observed in Linosa, where the seropositivity for $D$. immitis was higher than that of $D$. repens, most likely associated to the hyperendemicity of $D$. immitis in dogs from this island.

The circulation of Dirofilaria spp. in humans was also confirmed by the molecular detection of $D$. immitis in two samples from Linosa and one sample each for $D$. immitis and $D$. repens from Lampedusa. The molecular detection of $D$. immitis in human blood can be regarded as unusual considering that humans are dead-end hosts for dirofilarial infection with around 30 cases of patients, with or without clinical signs, detected positive at molecular or morphological isolation and identification of the parasite (Pampiglione et al., 2009; Genchi \& Kramer, 2020). The molecular positivity to $D$. immitis could be due to the presence of somatic material of worms in the peripheral bloodstream (Latrofa et al., 2012). In addition, since humans are dead-end hosts for $D$. immitis, chances of finding the microfilaria in peripheral circulation is low or null (Simón et al., 2012). Therefore, the possible reason for the positivity of D. immitis at blood examination is the highly sensitive of the qPCR test that could detect as few as a single microfilaria (Fink et al., 2011).

Conversely, D. repens may develop to adults in humans (Kramer et al., 2007; Poppert et al., 2009; Damle et al., 2014) occasionally producing microfilaraemia (Simón et al., 2012). This may be the reason of molecular detection of $D$. repens in sampled humans also by cPCR. Undoubtedly, the use of more invasive procedures, such as biopsies, and professional skills needed for the accurate morphological identification of the worm after biopsy makes the diagnosis of dirofilariasis a complex task (Simón et al., 2012). Under the above circumstances, the endosymbiont Wolbachia screening may also serve as an alternative/additional method for the diagnosis of dirofilariasis. Filarioids use Wolbachia for embryogenesis, development, fertility and longevity of the adult worm (Bandi et al., 1998; Simón et al., 2012; Lustigman et al., 2014; Manoj et al., 2021). This endosymbiont is released into the circulation and stimulate the host immune system, producing inflammation and immune response (Simón et al., 2012; Lustigman et al., 2014). However, the presence of Wolbachia in six human blood samples may also be associated with other filarioids of zoonotic importance (e.g., Onchocerca lupi), though it is highly improbable in the studied contexts. Hence, in areas where many filarioids occur in sympathry it is advisable to use more than one diagnostic methods, such as serum analysis and Wolbachia detection. Phylogenetic analyses of Wolbachia detected from D. repens positive human placed it along with other $\mathrm{C}$ group filarioids, especially with those of $D$. repens in a monophyletic clade, suggesting the importance of this endosymbiont in diagnosis.

Irrespective of the diagnostic methods used, molecular positivity to D. immitis in blood of humans further suggests the raising concern of active human infection with this species of filarioid, which can be misdiagnosed as D. repens (Foissac et al., 2013), being the latter considered the main causative agent of human dirofilariasis in Italy (Pampiglione et al., 2001; Gabrielli et al., 2021). Though, D. immitis has been related to nodules in lungs with no clinical sings (Avellis et al., 2011) dermatological findings (i.e., skin nodules, edema, subcutaneous lesions) and ocular lesions have also been found (Avellis et al., 2011; Foissac et al., 2013). Moreover, the serological results overlap with the most prevalent species of Dirofilaria in dogs for each island (i.e., D. repens in Lampedusa and $D$. immitis in Linosa), which is further confirmed by the molecular data. Nonetheless, the potential cross-reactivity of serological tests advocate for an integrative diagnosis with a morpho-molecular approach given that sole serological test can be misleading or less specific/sensitive.

\section{Conclusion}

Overall, the present study demonstrates the human exposure to D. immitis and D. repens in Linosa and Lampedusa islands. Particularly, the presence of higher seroprevalence of $D$. immitis in Linosa could be due to the hyperendemic status for canine heartworm demonstrated in a previous study (Brianti et al., 2021). The molecular confirmation of Dirofilaria spp. in 4 human samples also demonstrates that these parasites are currently circulating among dogs, people, and insect vectors in the study area. Herein, an integrative approach including the detection of Wolbachia endosymbionts is suggested as an alternative/additional diagnostic method for dirofilariasis. This is the first study reporting these parasites and their Wolbachia endosymbionts in humans from Linosa and Lampedusa, advocating measures of prevention and control regarding canine and human population in these islands.

\section{Author contribution}

JAMR: Conceptualization; Data curation; Formal analysis; Investigation; Methodology; Roles/Writing - original draft; Writing - review \& editing. SG: Data curation; Formal analysis; Investigation; Methodology; Writing - review \& editing. AC: Data curation; Investigation; Methodology; Writing - review \& editing. RRSM: Methodology; Roles/Writing original draft; Writing - review \& editing. MABS: Formal analysis; Roles/Writing - original draft; Writing - review \& editing. GB, EB: Formal analysis; Writing - review \& editing. MSL: Methodology; Writing 


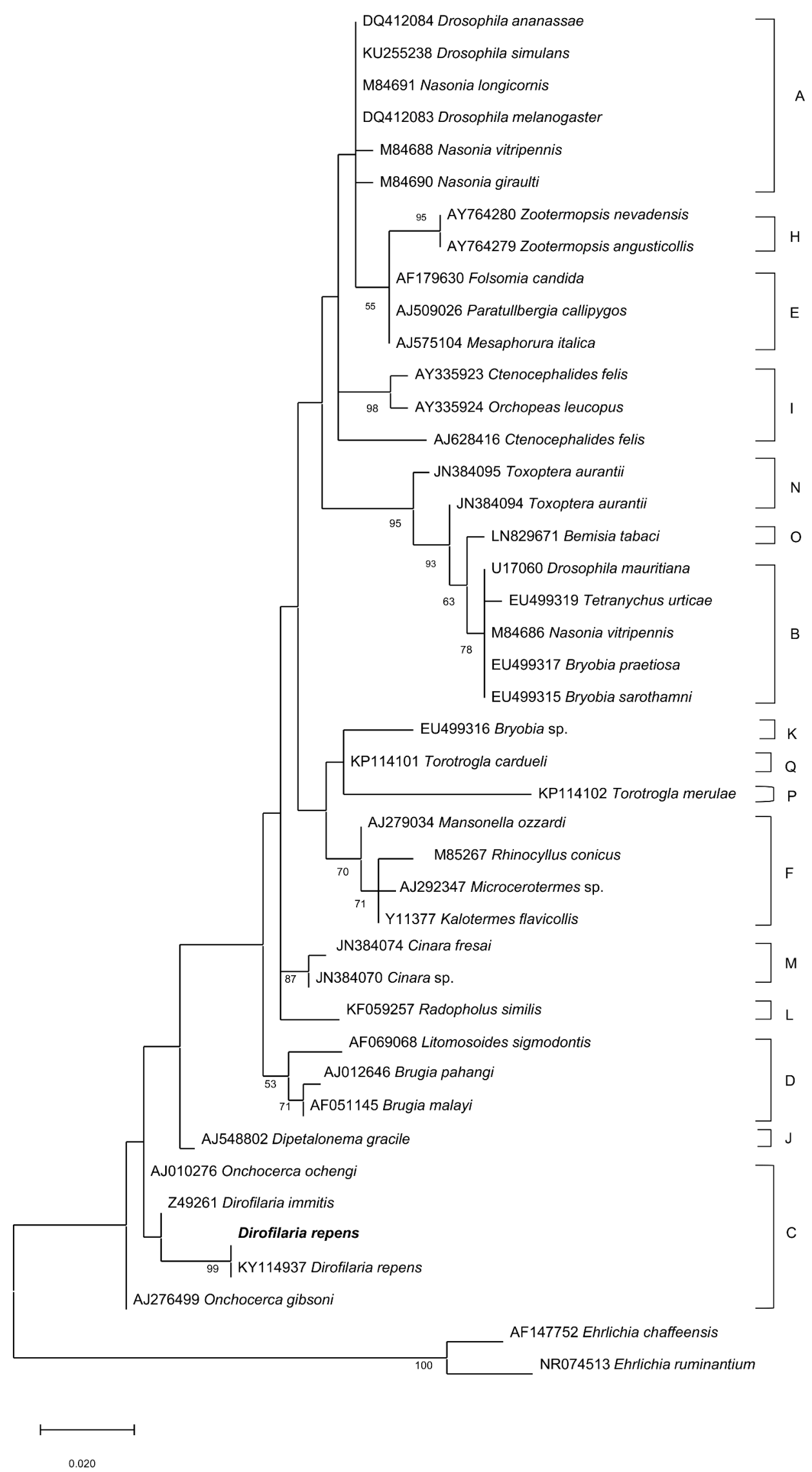$$
\text { (1) }
$$

Fig. 2. Phylogenetic relationship of Wolbachia detected in this study (in bold) and other available from GenBank belonging to different supergroups based on a partial sequence of the $16 \mathrm{~S}$ rRNA gene. Evolutionary analysis was conducted on 1000 bootstrap replications using Maximum Likelihood method and Kimura 2-parameter model with discrete Gamma distribution $(+G)$ to model evolutionary rate differences among sites selected by best-fit model. GenBank accession number and host species are indicated.

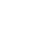


- review \& editing. DO: Conceptualization; Data curation; Formal analysis; Investigation; Project administration; Supervision; Validation; Visualization; Roles/Writing - original draft; Writing - review \& editing.

\section{Declaration of interests}

The authors declare that they have no known competing financial interests or personal relationships that could have appeared to influence the work reported in this paper.

\section{Acknowledgments}

The Authors would like to thank Giada Annoscia, Viviana Domenica Tarallo (Dipartimento di Medicina Veterinaria, Università degli Studi di Bari, Bari, Italy) for their support during the laboratory work, Irene Cambera (Marine biologist, Linosa) for her support during the field activities and Lorena Zecca (Dipartimento di Sanità Pubblica e Malattie Infettive, "Sapienza" Università di Roma) for the support during serological assays.

\section{References}

Avellis, F.O., Kramer, L.H., Mora, P., Bartolino, A., Benedetti, P., Rivasi, F., 2011. A case of human conjunctival dirofilariosis by Dirofilaria immitis in Italy. Vector Borne Zoonotic Dis. 11, 451-452. https://doi.org/10.1089/vbz.2010.0067.

Bandi, C., Anderson, T.J.C., Genchi, C., Blaxter, M.L., 1998. Phylogeny of Wolbachia in filarial nematodes. Proc. Biol Sci 265, 2407-2413. https://doi.org/10.1098/ rspb.1998.0591.

Bradford, M.M., 1976. A rapid and sensitive method for the quantitation of microgram quantities of protein utilizing the principle of protein-Dye binding. Anal. Biochem. 72, 248-254. https://doi.org/10.1006/abio.1976.9999.

Brianti, E., Panarese, R., Napoli, E., De Benedetto, G., Gaglio, G., Bezerra-Santos, M.A., Mendoza-Roldan, J.A., Otranto, D., 2021. Dirofilaria immitis infection in the Pelagie archipelago: the southernmost hyperendemic focus in Europe. Transbound Emerg Dis. https://doi.org/10.1111/tbed.14089.

Cabrera, E.D., Carretón, E., Morchón, R., Falcón-Cordón, Y., Falcón-Cordón, S., Simón, F., Montoya-Alonso, J.A., 2018. The Canary Islands as a model of risk of pulmonary dirofilariasis in a hyperendemic area. Parasitol. Res. 117, 933-936. https://doi.org/10.1007/s00436-018-5774-1.

Ciuca, L., Simòn, F., Rinaldi, L., Kramer, L., Genchi, M., Cringoli, G., Acatrinei, D., Miron, L., Morchon, R., 2018. Seroepidemiological survey of human exposure to Dirofilaria spp. in Romania and Moldova. Acta Trop. 187, 169-174. https://doi.org/ 10.1016/j.actatropica.2018.07.012.

Damle, A.S., Iravane, J.A., Khaparkhuntikar, M.N., Maher, G.T., Patil, R.V., 2014. Microfilaria in human subcutaneous dirofilariasis: a case report. J. Clin. Diag. Res.: JCDR 8, 113. https://doi.org/10.7860/JCDR/2013/6886.4123.

Dantas-Torres, F., Otranto, D., 2020. Overview on Dirofilaria immitis in the Americas, with notes on other filarial worms infecting dogs. Vet. Parasitol. 282, 109113 https://doi.org/10.1016/j.vetpar.2020.109113.

Esteban-Mendoza, M.V., Arcila-Quiceno, V., Albarracín-Navas, J., Hernández, I., FlechasAlarcón, M.C., Morchón, R., 2020. Current situation of the presence of Dirofilaria immitis in dogs and humans in bucaramanga, Colombia. Front Vet Sci. 7, 488. https://doi.org/10.3389/fvets.2020.00488.

Fink, D.L., Kamgno, J., Nutman, T.B., 2011. Rapid molecular assays for specific detection and quantitation of Loa loa microfilaremia. PLoS Negl Trop Dis 5, e1299. https:// doi.org/10.1371/journal.pntd.0001299.

Foissac, M., Million, M., Mary, C., Dales, J.P., Souraud, J.B., Piarroux, R., Parola, P., 2013. Subcutaneous infection with Dirofilaria immitis nematode in human. France. Emerg Infect Dis. 19, 171-172. https://doi.org/10.3201/eid1901.120281.

Gabrielli, S., Mangano, V., Furzi, F., Oliva, A., Vita, S., Poscia, R., Fazii, P., Di Paolo, J., Marocco, R., Mastroianni, C.M., Bruschi, F., Mattiucci, S., 2021. Molecular identification of new cases of human Dirofilariosis (Dirofilaria repens) in Italy. Pathogens 10, 251. https://doi.org/10.3390/pathogens10020251.

Genchi, C., Kramer, L.H., 2020. The prevalence of Dirofilaria immitis and D. repens in the old world. Vet. Parasitol. 280, 108995 https://doi.org/10.1016/j. vetpar.2019.108995.

Kimura, M., 1980. A simple method for estimating evolutionary rates of base substitutions through comparative studies of nucleotide sequences. J. Mol. Evol. 16, $111-120$.

Kramer, L.H., Kartashev, V.V., Grandi, G., Morchón, R., Nagornii, S.A., Karanis, P., Simón, F., 2007. Human subcutaneous dirofilariasis, Russia. Emerg. Infect. Dis. 13, 150-152. https://doi.org/10.3201/eid1301.060920.
Kumar, S., Stecher, G., Li, M., Knyaz, C., Tamura, K., 2018. MEGA X: molecular evolutionary genetics analysis across computing platforms. Mol. Biol. Evol. 35, 1547-1549. https://doi.org/10.1093/molbev/msy096.

Latrofa, M.S., Dantas-Torres, F., Annoscia, G., Genchi, M., Traversa, D., Otranto, D., 2012. A duplex real-time polymerase chain reaction assay for the detection of and differentiation between Dirofilaria immitis and Dirofilaria repens in dogs and mosquitoes. Vet. Parasitol. 185, 181-185. https://doi.org/10.1016/j. vetpar.2011.10.038.

Lustigman, S., Melnikow, E., Anand, S.B., Contreras, A., Nandi, V., Liu, J., Bell, A., Unnasch, T.R., Rogers, M.B., Ghedin, E., 2014. Potential involvement of Brugia malayi cysteine proteases in the maintenance of the endosymbiotic relationship with Wolbachia. Int. J. Parasitol. Drugs Drug Resist. 4, 267-277. https://doi.org/10.1016/ j.ijpddr.2014.08.001.

Manoj, R.R.S., Latrofa, M.S., Epis, S., Otranto, D., 2021. Wolbachia: endosymbiont of onchocercid nematodes and their vectors. Parasit. Vector. 14, 245. https://doi.org/ 10.1186/s13071-021-04742-1.

Mendoza-Roldan, J., Benelli, G., Panarese, R., Iatta, R., Furlanello, T., Beugnet, F., Zatelli, A., Otranto, D., 2020. Leishmania infantum and Dirofilaria immitis infections in Italy, 2009-2019: changing distribution patterns. Parasit Vectors 13, 193. https:// doi.org/10.1186/s13071-020-04063-9.

Oliva, A., Gabrielli, S., Pernazza, A., Pagini, A., Daralioti, T., Mantovani, S., Mattiucci, S., D'Amati, G., Mastroianni, C.M., 2019. Dirofilaria repens infection mimicking lung melanoma metastasis. Open Forum Infect. Dis. 6, ofz049. https://doi.org/10.1093/ ofid/ofz049.

Otranto, D., Brianti, E., Dantas-Torres, F., Weigl, S., Latrofa, M.S., Gaglio, G., Cauquil, L., Giannetto, S., Bain, O., 2011. Morphological and molecular data on the dermal microfilariae of a species of Cercopithifilaria from a dog in Sicily. Vet. Parasitol. 182, 221-229. https://doi.org/10.1016/j.vetpar.2011.05.043.

Otranto, D., Dantas-Torres, F., Brianti, E., Traversa, D., Petrić, D., Genchi, C., Capelli, G., 2013. Vector-borne helminths of dogs and humans in Europe. Parasit. Vectors 6, 16. https://doi.org/10.1186/1756-3305-6-16.

Pampiglione, S., Rivasi, F., Angeli, G., Boldorini, R., Incensati, R.M., Pastormerlo, M., Pavesi, M., Ramponi, A., 2001. Dirofilariasis due to Dirofilaria repens in Italy, an emergent zoonosis: report of 60 new cases. Histopathology 38, 344-354. https://doi. org/10.1046/j.1365-2559.2001.01099.x.

Panarese, R., Iatta, R., Latrofa, M.S., Zatelli, A., Ćupina, A.I., Montarsi, F., Pombi, M., Mendoza-Roldan, J.A., Beugnet, F., Otranto, D., 2020. Hyperendemic Dirofilaria immitis infection in a sheltered dog population: an expanding threat in the Mediterranean region. Int. J. Parasitol. 50, 555-559. https://doi.org/10.1016/j. ijpara.2020.04.002.

Poppert, S., Hodapp, M., Krueger, A., Hegasy, G., Niesen, W.D., Kern, W.V., Tannich, E., 2009. Dirofilaria repens infection and concomitant meningoencephalitis. Emerg. Infec. Dis. 15, 1844-1846. https://doi.org/10.3201/eid1511.090936.

Prieto, C., Venco, L., Simon, F., Genchi, C, 1997. Feline heartworm (Dirofilaria immitis) infection: detection of specific IgG for the diagnosis of occult infections. Vet. Parasitol. 70, 209-217. https://doi.org/10.1016/s0304-4017(97)00008-3.

Simón, F., Siles-Lucas, M., Morchón, R., González-Miguel, J., Mellado, I., Carretón, E., Montoya-Alonso, J.A., 2012. Human and animal dirofilariasis: the emergence of a zoonotic mosaic. Clin. Microbiol. Rev. 25, 507-544. https://doi.org/10.1128/ CMR.00012-12.

Tamura, K., Stecher, G., Peterson, D., Filipski, A., Kumar, S., 2013. Molecular evolutionary genetics analysis version 6.0. Mol. Biol. Evol. 30, 2725-2729. https:// doi.org/10.1093/molbev/mst197.

Tasić-Otašević, S.A., Gabrielli, S.V., Tasić, A.V., Miladinovićtasić, N.L., Kostić, J.T., Ignjatović, A.M., Tasić-Otašević, S.A., Trenkić Božinović, M.S., Gabrielli, S.V., Genchi, C., 2015. Canine and human Dirofilaria infections in the Balkan Peninsula. Vet. Parasitol. 209, 151-156. https://doi.org/10.1016/j.vetpar.2015.02.016.

Tasić-Otašević, S.A., Gabrielli, S.V., Tasić, A.V., Miladinovićtasić, N.L., Kostić, J.T., Ignjatović, A.M., Popović Dragonjić, L.D., Milošević, Z.G., Arsić-Arsenijević, V.S., Cancrini, G.A., 2014. Seroreactivity to Dirofilaria antigens in people from different areas of Serbia. BMC Infect. Dis. 14, 68. https://doi.org/10.1186/1471-2334-14-68.

Turba, M.E., Zambon, E., Zannoni, A., Russo, S., Gentilini, F., 2012. Detection of Wolbachia DNA in blood for diagnosing filaria-associated syndromes in cats. J. Clin. Microbiol. 50, 2624-2630. https://doi.org/10.1128/JCM.00528-12.

Werren, J.H., Windsor, D.M., 2000. Wolbachia infection frequencies in insects: evidence of a global equilibrium? Proc. Biol. Sci. 267, 1277-1285. https://doi.org/10.1098/ rspb.2000.1139.

Willems, A., Watteyne, S., Mertens, J., Gillis, M., Vandekerckhove, T.T.M., Swing, J.G., 1999. Phylogenetic analysis of the $16 \mathrm{~S}$ rDNA of the cytoplasmic bacterium Wolbachia from the novel host Folsomia candida (Hexapoda, Collembola) and its implications for Wolbachial taxonomy. FEMS Microbiol. Lett. 180, 279-286. https://doi.org/ 10.1111/j.1574-6968.1999.tb08807.x.

Zumaquero, L., Simón, F., Carretón, E., Hernández, I., Sandoval, C., Morchón, R., 2020. Prevalence of canine and human dirofilariosis in Puebla. Mexico. Vet Parasitol. 282, 109098 https://doi.org/10.1016/j.vetpar.2020.109098. 